\title{
Discussion on Evaluation Method of Helium Resources in Natural Gas
}

\author{
Zhang Zuoxiang, Jin Dawei, Bai Fengyou, Wang Yujie, Dong Juan, Chen Yan, Gu Qinglian \\ Daqing Oilfield Exploration and Development Research Institute, Daqing, China \\ Email address: \\ zuoxiangzhang@petrochina.com.cn (Zhang Zuoxiang),jindawei@petrochina.com.cn (Jin Dawei), baify@petrochina.com.cn (Bai Fengyou), \\ yujiewang@petrochina.com.cn (Wang Yujie),dongjuan@petrochina.com.cn (Dong Juan), gy_chenyan1@petrochina.com.cn (Chen Yan), \\ guqinglian@petrochina.com.cn (Gu Qinglian)
}

\section{To cite this article:}

Zhang Zuoxiang, Jin Dawei, Bai Fengyou, Wang Yujie, Dong Juan, Chen Yan, Gu Qinglian. Discussion on Evaluation Method of Helium Resources in Natural Gas. Science Discovery. Vol. 6, No. 1, 2018, pp. 1-5. doi: 10.11648/j.sd.20180601.11

Received: January 19, 2018; Accepted: February 5, 2018; Published: March 9, 2018

\begin{abstract}
In view of the importance of helium resources and the lack of evaluation methods, the resource evaluation method for helium in natural gas is found and summarized. Helium gas is associated with oil and gas, and generally exists as a component of natural gas. It has important industrial value and is used in many fields. At present, the helium resources are scarce in China, the application of the extraction of helium gas from natural gas is less. Based on sorting and summarizing the evaluation methods of natural gas, choose the resource evaluation method that can be applied to helium, and evaluate the resource of helium in natural gas based on the natural gas evaluation method. Finally, select the target area to select the evaluation parameters and control the reserves calculation, to verify the feasibility of the method. Through verification, the volumetric method is an accurate and reliable evaluation method for the helium gas resources in natural gas.
\end{abstract}

Keywords: Natural Gas, Helium, Resources, Evaluation

\section{天然气中氦资源评价方法探讨}

\section{张作祥, 金大伟, 白凤有, 王玉杰, 董娟, 陈䎦, 顾青莲}

勘探开发研究院, 大庆油田有限责任公司, 大庆, 中国

\section{邮箱}

zuoxiangzhang@petrochina.com.cn（张作祥）, jindawei@petrochina.com.cn（金大伟）, baify@petrochina.com.cn（白凤有）， yujiewang@petrochina.com.cn（王玉杰）, dongjuan@petrochina.com.cn（董娟）, gy_chenyan1@petrochina.com.cn（陈艳）, guqinglian@petrochina.com.cn (顾青莲)

摘要: 针对氦资源重要性和评价方法的缺失, 寻找和总结出适用于天然气中氦的资源评价方法。氦气与油气伴生, 一 般作为天然气中的一种组分而存在, 其具有重要的工业价值, 应用于众多领域。中国目前氦气资源匮乏, 而且天然气 中氦气资源应用较少。力求在整理和总结天然气评价方法的基础上, 来选取可适用于氦气的资源评价方法, 并基于天 然气评价方法进行天然气中氦的资源评价。最后, 选取目标区进行评价参数的选取和控制储量计算, 来验证方法的可 行性。经过验证，容积法是评价天然气中氦气资源的准确可靠评价方法。

关键词: 天然气, 氦, 资源, 评价 


\section{1. 引言}

氦具有重要的工业价值, 在工业和高科技领域中有广 阔的应用前景 [1], 例如国防军工、航空航天工业、医 学医药、超导技术、深水潜水、激光、工业分析、环境 控制等等。但是, 中国的氦资源却相对稀缺, 发现的氦 气资源数量较少, 资源量不到世界总资源量的 $0.1 \%$ $0.2 \%[2]$ 。目前中国使用的氦气主要依靠进口，价格相 当昂贵。因此, 对天然气中的氦进行资源评价研究具有 重要意义。

\section{2. 氦的性质及成藏条件}

氦气是一种惰性气体, 也是稀有资源。在通常情况下 为无色、无臭、无味。氦由原子量为 4.003 的 ${ }^{4} \mathrm{He}$ 和原子量 为3.016的 ${ }^{3} \mathrm{He}$ 这两种稳定的同位素组成, 是所有元素中最 不活泼的元素, 极难形成化合物, 具有不易液化、稳定性 好、扩散性强等特点[3]。

大量的研究表明 [4-5], 天然气中的氦都是无机成因的, 包括壳源成因和幔源成因两种。在氦的同位素上, ${ }^{3} \mathrm{He}$ 主 要是元素合成时形成的核素, 即是地球形成过程中捕获的 原始氦, ${ }^{4} \mathrm{He}$ 则主要是地球中放射性元素铀和针元素衰变 所产生, 因此壳源氦和幔源氦的 ${ }^{3} \mathrm{He} /{ }^{4} \mathrm{He}$ 比值差别较大。 通常以 $\mathrm{R}={ }^{\mathrm{w}}\left({ }^{3} \mathrm{He}\right) / \mathrm{w}\left({ }^{4} \mathrm{He}\right)$ 来表示氦的来源, 其中 $\mathrm{R}$ 值为 $2.0 \times 10^{-8}$ 和 $1.1 \times 10^{-5}$ 时, 分别表示壳源同位素特征值和幔源 同位素特征值, 大气中的氦是两种成因氦混合的结果, ${ }^{3} \mathrm{He}$ $/^{4} \mathrm{He}$ 值为 $1.4 \times 10^{-6}$ 。也常用样品氦 $(\mathrm{R})$ 和大气氦 $(\mathrm{Ra})$ 的同位 素比值来表示气样的氦同位素特征[6-7], 即 $\mathrm{R} / \mathrm{Ra}=\left(\mathrm{w}_{3 \mathrm{He}}\right.$ $/ \mathrm{W}_{4 \mathrm{He}}$ )䍺 $/\left(1.4 \times 10^{-6}\right)$, 当 $\mathrm{R} / \mathrm{Ra}<1$ 时, 表示为壳源; $\mathrm{R} / \mathrm{Ra}>1$ 时, 表示为幔源[6-8]。

地层中单独的氦气藏很罕见, 主要以油气的伴生物为 主, 但是与油气的生成没有直接联系。因此, 在常规产出 的天然气中, 一般会伴有含量不等的氦气，这种特点为氦 气资源的获取和开发提供了潜在领域。

氦气一般与油气伴生, 作为天然气的一种组分而存在, 因此其成藏过程与天然气的成藏过程基本一致。又因为氦气 的分子量小于烷烃气的分子量, 其渗透性要强于常规天然气 组分, 更容易通过盖层和运移通道而散失。在成藏过程中一 般会处于不断补给和不断散失的动平衡过程中, 因此氦气成 藏的条件相比其他天然气组分更加的苛刻, 不仅需要良好的 圈闭条件以及更加致密和封闭的盖层条件, 还需要动态的、 不断的氦气补给, 以满足补给量大于散失量的条件。因此, 丰富的氦气源、良好的圈闭、致密的盖层、畅通的气源运移 通道和不断的氦气补给是氦气成藏的重要条件。

\section{3. 天然气中氦气资源评价方法}

现今用于石油、天然气资源定量评价的方法数量众多, 需要针对某一油气藏的勘探程度、地质特征、驱动类型 等实际情况来选择合适的评价方法。目前国内外常用的 油、气藏资源定量评价主要包括类比法、容积法、物质
平衡法、产量递减法、矿场不稳定试井法、水驱特征曲 线法、统计模拟法 (又称蒙特卡洛法 (Monte-Carlo) 或概率统计法)、外推预测法、灰色预测法、油田规模 序列法等。

由于氦气与天然气的伴生关系, 因此进行天然气中氦 气资源的定量评价, 所需采用的方法一般基于天然气评 价方法。需要在选取常规的油气藏评价方法的基础上, 结合该气藏氦气的含量、分布特点、地质特征等情况来 进行天然气中氦气资源评价。

\section{1. 容积法}

容积法是计算油、气藏地质储量的主要方法, 应用最广 泛。它适用于不同的勘探开发阶段、不同的圈闭类型、不同 的储集类型和驱动方式。容积法计算结果的可靠程度取决于 资料数量和质量。对于大、中型构造砂岩储集层油、气藏, 计算精度较高, 而对于复杂类型油、气藏, 则准确性较低。 容积法计算天然气中氦的公式 (1)：

$$
\mathrm{G}_{\mathrm{He}}=\mathrm{He} \cdot 0.01 \mathrm{Ag} \cdot \mathrm{h} \cdot \Phi \cdot \mathrm{Sg} \cdot \mathrm{Tsc} \cdot \mathrm{Pi} /(\mathrm{Psc} \cdot \mathrm{T} \cdot \mathrm{Zi})
$$

式中:

$G_{H e}$ 一天然气中氦气的探明地质储量, $10^{8} \mathrm{~m}^{3}$;

$\mathrm{He}$-天然气中氦气的含量, $\%$;

$\mathrm{Ag}$ 一含气面积, $\mathrm{km}^{2}$;

$h$ 一有效厚度, $\mathrm{m}$;

$\Phi$ 一平均有效孔隙度, $\%$;

$S g$ 一平均原始含气饱和度, $\%$;

$T$-气层温度, $\mathrm{K}$;

$T s c$ 一地面标准温度, K;

$P s c$ 一地面标准压力, $\mathrm{MPa}$;

$\mathrm{Zi}$ 一原始气体偏差系数, 无因次量;

$P i$-气藏的原始地层压力, $\mathrm{MPa}$ 。

\section{2. 蒙特卡罗法}

蒙特卡罗法是以概率论与数理统计理论为指导, 有着广 泛应用领域的通用性统计学方法。目前世界上各主要产油 国及西方各大石油公司都把这种方法作为石油资源量定量 评价的重要方法之一, 广泛应用于各含油气区的早中期勘探 阶段。1979 年中国把蒙特卡罗法用于油气资源评价, 目前 是中国以统计预测为主的资源评价的核心方法之一。

利用蒙特卡洛法进行储量估算的氦气资源量计算公 式如式 (2):

$$
\mathrm{Q}_{\mathrm{He}}=\mathrm{He} \cdot \mathrm{S} \cdot \mathrm{H} \cdot \varphi \cdot \mathrm{C} \cdot \gamma \cdot \mathrm{B}
$$

式中:

$Q_{H e}$ 一地质圈闭的氦气资源量, $\mathrm{t}$;

$\mathrm{He}$-天然气中氦气的含量, $\%$;

$S$ 一地质圈闭的分布面积, $\mathrm{Km}^{2}$;

$H$ 一储气层厚度, $\mathrm{km}$;

$\varphi$ 一储气层孔隙度, $\%$;

$C$ 一含气饱和度, $\%$; 
$\gamma$ 一天然气比重, $\mathrm{Kg} / \mathrm{m}^{3}$;

$B$ 一体积系数。

\section{3. 残烃法}

残烃法是中国应用比较广泛的一种资源量计算方法, 在残烃法中常用的是氯仿沥青“A”法, 它是以生油层系的 残留氯仿沥青“A”抽提量为基础的方法, 其计算公式为式 (3):

$$
\mathrm{Q}_{\mathrm{He}}=\mathrm{He} \cdot \frac{1}{1-k_{\text {排 }}} \mathrm{S} \cdot \mathrm{H} \cdot \text { “A” } \mathrm{d} \cdot \mathrm{k}^{\text {聚 }}
$$

式中:

$Q_{H e}$ 一氦气总资源量, $\mathrm{t}$;

$\mathrm{He}$-天然气中氦气的含量, \%;

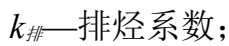

$S$ 一有效生油岩面积, $\mathrm{km}^{2}$;

$H$ 一有效生油岩厚度, $\mathrm{km}$;

“ $A$ ”一氯仿沥青“A”含量, \%;

$d$ 一生油岩密度, $\mathrm{t} / \mathrm{km}^{3}$;

$k$ 聚一聚集系数。

\section{4. 热模拟法}

邬立言等建立的快速定量评价方法认为, 一个盆地或 地区只要做一定数量的热解分析就可以根据分析结果定 出有机质类型、产烃率、降解率, 进而计算出盆地的生油 气量和总资源量。此方法同样也适用于本地区。其具体工 作公式如式 (4)：

$$
\mathrm{Q}_{\mathrm{He}}=\mathrm{He} \mathrm{C}_{\text {原 }} \cdot \mathrm{D} / 0.083 \cdot \mathrm{H} \cdot \mathrm{S} \cdot \rho \cdot \mathrm{K}_{\text {排 }} \cdot \mathrm{K}_{\text {聚 }}
$$

式中:

$Q_{H e}$ 一氦气总资源量, $\mathrm{m}^{3}$;

$\mathrm{He}$-天然气中氦气的含量, $\%$;

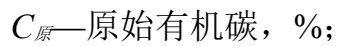

$D$ 一成熟生油岩的降解率，\%;

0.083 一从碳换算为烃量 $\mathrm{kg} / \mathrm{t}$ 的换算系数;

$H$ 一计算点的生油岩厚度, $\mathrm{km}$;

$S$ 一生油岩面积, $\mathrm{km}^{2}$;

$\rho$ 一生油岩密度, $\mathrm{t} / \mathrm{km}^{3}$;

K排一排烃系数;

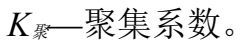

\section{5. 灰色系统预测法}

灰色理论是由华中理工大学邓聚龙教授于 1982 年 首次提出的, 并且己陆续用于农业、经济、科技、社会 等领域, 取得了应有的效果。灰色系统理论是研究本征 性灰色系统的量化问题, 是研究系统的建模、预测、分 析、决策和控制的理论。按照人们对信息系统的知晓程 度, 通常可以将信息系统分为三类, 即白色系统、灰色 系统和黑色系统, 当一种信息系统完全未知时, 称其为 黑色系统, 如距地球遥远的星球, 只知它一定存在, 但 它距地球多远, 质量、体积是多少等等都不知道, 所以 就属于黑色系统; 反之, 当一种系统是完全已知时, 称
其为白色系统; 而介于两者之间的, 既含有白色信息, 又含有黑色信息的系统就叫灰色系统。灰色系统理论认 为对既含有已知信息又含有未知或非确定信息的系统 进行预测, 就是对在一定方位内变化的、与时间有关的 灰色过程的预测。尽管过程中所显示的现象是随机的、 杂乱无章的, 但毕竟是有序的、有界的, 因此这一数据 集合具备潜在的规律, 灰色预测就是利用这种规律建立 灰色模型对灰色系统进行预测。

\section{6. 油田规模序列法}

国内外许多含油气区的统计资料表明, 当一个含油气 区的一些油气田被发现后, 如果以油田规模为纵坐标, 油 田规模序号为横坐标, 在双对数坐标纸上展点作图大致可 得到一条直线。根据这一规律, 可以在探区的早期或中期 勘探阶段, 由已发现的油田规模序列, 预测尚未发现的油 气田储量以及整个探区的油气总储量, 这种方法称为油田 规模序列法。

\section{7. 动态法}

难以容积法计算地质储量时, 可以采用动态法计算, 根据产量、压力数据的可靠程度, 划分探明地质储量和控 制地质储量。

天然气藏主要采用物质平衡法和弹性二相法计算天 然气地质储量, 再根据计算结果来评价氦气资源。

（1）物质平衡法: 采用物质平衡法的压降图（视地 层压力与累积产量关系图) 直线外推法, 废弃视地层压力 为零时的累积产量即为天然气地质储量。

（2）弹性二相法: 采用井底流动压力与开井生产时 间的压降曲线图直线段外推法, 废弃相对压力为零时可计 算单井控制的天然气地质储量。

\section{4. 实例计算}

选取了 S3区块进行天然气中氦的资源评价, 根据目前 对该区的地质认识程度和勘探程度, 决定采用容积法计算 该地区的天然气中氦气的控制储量。计算参数选择及计算 结果如下:

\section{1. 含气面积}

圈定含油（气）面积, 需充分利用地震、钻井、测井 和测试 (含试油) 等资料, 综合研究油、气、水分布规律 和油 (气) 藏类型, 确定流体界面 (即气油界面、油水界 面、气水界面）以及油气遮挡（如断层、岩性、地层）边 界, 编制反映油气层 (储集体) 顶（底）面形态的海拔高 度等值线图。

选定研究区, 在储层预测结果的基础上, 根据井点气 水界面及气藏认识编绘有效厚度等值图, 根据井点外推及 有效厚度分布趋势分别圈定含气面积。不同类别的地质储 量, 含油 (气) 面积圈定要求不同。

其中 S3区块控制储量含气面积圈定原则: 井点外推 小于 $3 \mathrm{~km}$, 对于有效厚度大的可局部放宽; 有效厚度大 于 $20 \mathrm{~m}$ 。 


\section{2. 有效厚度}

油（气）层有效厚度（简称有效厚度）, 指达到储量 起算标准的含油气层系中具有产油气能力的那部分储层 厚度。不同类别的地质储量, 有效厚度确定要求不同。

\subsection{1. 有效厚度下限标准}

由于本区储层物性较差, 同时还含有 $\mathrm{CO}_{2}$ 气, 因此未 压裂的干层与含 $\mathrm{CO}_{2}$ 气层均未计入误差范围。判别结果有 误的气水同层点 2 层、干层1层, 图版精度 $91.90 \%$ （表1）。

表1 目标区块有效厚度下限标准。

\begin{tabular}{lllllll}
\hline \multirow{2}{*}{ 层位 } & 气层 & 电性 & & & 扣夹层 \\
\cline { 3 - 6 } & 类别 & 电阻率 $(\boldsymbol{\Omega m})$ & 声波时差 $(\boldsymbol{\mu} \mathbf{s} / \mathbf{m})$ & 气测比值 & 密度 $\left(\mathbf{g} / \mathbf{c m}^{3}\right)$ & \\
\hline \multirow{2}{*}{$\mathrm{D} 1$} & 气层 & $\mathrm{LLD} \geq 110$ & $\mathrm{DT} \geq 55$ & $\geq 2.6$ & $\leq 2.53$ & $\mathrm{DEN}>2.53$ \\
& & & & & & $\mathrm{LLS}$ 回返程度 $\geq 25 \%$ \\
\hline
\end{tabular}

\subsection{2. 有效厚度取值}

利用上述标准划分了单井有效厚度, 以此标定地震, 预测了有效厚度分布。计算含气面积内平均有效厚度, S3 区块控制面积内有 3 口探井控制, 预测面积可靠性较高, 取平均有效厚度 $70 \%$ 为储量计算值。

\section{3. 有效孔隙度}

储量计算中所用的有效孔隙度是指有效厚度段的地 下有效孔隙度。有效孔隙度可直接用岩心分析资料, 也可 用测井解释确定。测井解释孔隙度与岩心分析孔隙度的相 对误差不超过 $\pm 8 \%$ 。裂缝孔隙型储层必须分别确定基质孔 隙度和裂缝、溶洞孔隙度。

目标区S3区块控制储量有效孔隙度采用 S1井和S2井 有效厚度加权计算并经压实校正, S3 区块预测储量有效孔 隙度分别参考探明储量计算值取值。

\section{4. 原始含气饱和度}

大型以上油（气）田（藏）用测井解释资料确定探明 储量含油（气）饱和度（\%) 时, 应有油基泥浆取心或密 闭取心分析验证，绝对误差不超过 $\pm 5 \%$ 。特殊情况除外。 中型以上油 (气) 田 (藏) 用测井解释资料确定含油 (气) 饱和度时, 应有实测的岩电实验数据及合理的地层水电阻 率资料。

在目标区利用核磁计算、常规测井计算与压录法三种 饱和度模型计算的 5 口井中, 核磁计算求得平均原始含气 饱和度为 $61.8 \%$, 常规测井计算结果为 $60.0 \%$, 压录法求
得平均原始含气饱和度为 $65.0 \%$ 。密闭取心分析最大含气 饱和度 $85.2 \%$, 最小含气饱和度 $64.2 \%$, 平均含气饱和度 $72.3 \%$ 。综合分析来看, 密闭取心结果与三种方法计算的 结果, 都有一定的局限性, 但总体上原始含气饱和度在 $60.0-72.3 \%$ 之间。

因此, 本次储量计算主要是依据各区块的储层物性情 况, 参考探明储量取值确定。

\section{5. 原始天然气体积系数}

原始天然气体积系数, 指原始地层条件下天然气体积 与地面标准条件下天然气体积的比值, 表达式为 $B g i=$ $P s c \cdot Z i \cdot T / P i \cdot T s c$ 。由于目标区块没有气分析数据, 因此, 本次储量计算临井数据推算。对 $\mathrm{S} 3$ 区块控制储量区, 利用 临井 $(\mathrm{S} 1$ 和 $\mathrm{S} 2)$ 的气藏中部深度推算该区中部深度后, 利 用 $\mathrm{S} 3$ 井的温度、压力梯度推算其压力、温度值。

\section{6. 天然气中氦气的含量}

目前, 天然气中氦的分析方法主要采用的是气相色谱 法, 其测试氦气的浓度范围一般是 $0.01 \% \sim 10 \%$ 。因此, 目标区天然气中氦的含量使用气相色谱法的测定。

\section{7. 计算}

根据容积法计算公式 (1) 进行计算, 具体计算结果 如下表 2 。该目标区天然气中氦气的含量为 $0.133 \%$, 控制 地质储量为 $0.63 \times 10^{8} \mathrm{~m}^{3}$ 。

表2 天然气中氦的控制地质储量数据表。

\begin{tabular}{|c|c|c|c|c|c|c|c|c|}
\hline 区块 & 层位 & $\begin{array}{l}\text { 含气面积 } \\
\mathbf{k m}^{2}\end{array}$ & $\begin{array}{l}\text { 有效厚度 } \\
\text { m }\end{array}$ & $\begin{array}{l}\text { 有效孔隙度 } \\
\%\end{array}$ & $\begin{array}{l}\text { 含气饱和度 } \\
\%\end{array}$ & 体积系数 & $\begin{array}{l}\text { 天然气中氦含量 } \\
\%\end{array}$ & $\begin{array}{l}\text { 控制地质储量 } \\
\mathbf{1 0}^{8} \mathbf{m}^{3}\end{array}$ \\
\hline S3 & D1 & 118.2 & 45.5 & 5.8 & 58.0 & 0.00383 & 0.133 & 0.63 \\
\hline
\end{tabular}

\section{5. 结论}

氦气资源评价方法主要是基于天然气资源评价方法 上的选择和改进, 目前合适的的方法主要有容积法、蒙特 卡罗法、残烃法、热模拟法等。

根据目标区的综合特征，选择了容积法对目标区的氦 气资源进行评价, 并进行了计算参数的选择。

目前, 含氦天然气仍是世界上氦气的主要来源。而 中国是氦气资源贫乏的国家, 只有四川自贡威远含氦天
然气(其含氦为 $0.18 \% \sim 0.20 \%$ )得到提取利用; 另外, 渭 河盆地、苏北盆地等开展了含氦天然气资源的远景评价。 因此, 天然气中的氦气资源对中国有十分重要的意义。 今后在进行天然气中氦气资源评价的基础上，寻找当前 的技术条件和经济条件下具有工业价值的氦气资源依 然是重中之重。另外，在具有氦气资源前景的地区的油 气藏勘探开发过程中, 加强对天然气组分中氦气的检测 与分析和天然气中氦气的提取技术研究也是十分迫切 且很有必要的。 


\section{参考文献}

[1] 史斗译.美国的氦气工业[J]. 天然气地球科学, 1991, 2(5): $245 \sim 248$

[2] 张子枢. 中国氦资源及其开发与保护 $[\mathrm{J}]$. 资源开发与保护, 1987, 3(4): 28〜31

[3] 李广之, 高伟, 江浩, 等. 氦气的天然气地质意义 $[\mathrm{J}]$. 物探 与化探, 2009.33(2):154 156

[4] Mamyrin. B. A, Tolstikhin IN. Helium isotopes in nature. Amsterdam[J], Elsevier, 1984: 175 179

[5] Kaneoka I, Takakka N. Noble-g as state in the Earth's interiorsome constaints on the present state[J]. Chemical Geology(Iso tope Geoscience section), 1985, 52: 75 $\sim 95$

[6] 徐永昌. 天然气中氦同位素分布及构造环境[J]. 地学前缘, 1997(z2): 189 194

[7] 杨春, 陶士振, 侯连华, 等. 松辽盆地火山岩储层天然气 藏He同位素组成累积效应 [J]. 天然气地球科学, 2014, 25(1):109-115

[8] 戴金星, 石昕, 卫延召. 无机成因油气论和无机成因的气 田(藏)概略 [J].石油学报，2001，22 (6)：5 10

[9] 余琪祥，史政等. 塔里木盆地西北部氦气富集特征与成藏 条件分析[J]. 西部地质, 2013,46（4）:215 222

[10] 李玉宏, 王行运, 韩伟. 渭河盆地氦气资源远景调查进展 与成果 $[\mathrm{J}]$.中国地质调查,2015,2(6):1 6

[11] 韩伟, 李玉宏, 卢进才, 等. 陕西渭河盆地富氦天然气异 常的影响因素 $[J]$. 地质通报, 2014，33(11):1836 1841

[12] 张福礼, 孙启邦, 王行运, 等. 渭河盆地水溶氦气资源评 价[J]. 地质力学学报, 2012, 18(2):195 202

[13] 丁涛.苏北地区含氦天然气资源综合利用前景展望 [J]. 内蒙 古石油化工，2010，36 (17): 64-64

[14] 陶成, 刘文汇, 等. 天然气藏 $\mathrm{H}$ e 的累积模式及定年应用初 探 $[J]$. 地质学报, 2015 (7) :1302 1307

[15] 张亮亮, 孙庆国.等. LNG尾气中提取氦气的流程分析 [J]. 低 温技术, $2015,43(2): 29 \sim 33$

[16] 张亮亮, 孙庆国, 等. 氦气全球市场及中国氦气安全保障的 建议[J].低温与特气，2014,32（3）:1〜5 